\title{
冠醚手性固定相的合成及其性能评价
}

\author{
路振宇伍鹏字敏杨璨瑜 孔娇袁黎明* \\ (云南师范大学化学化工学院 昆明 650500)
}

\begin{abstract}
摘要 以 $R$-联菜酚为原料, 利用铃木反应改进合成了已有文献报道的 $R$-(3,3'-二苯基-1,1'-二荎基)-20-冠-6, 并将其涂敷 于 $\mathrm{C}^{18}$ 硅胶(平均粒径 $5 \mu \mathrm{m}$, 孔径 $120 \AA$ )上制成了可用于高效液相色谱手性拆分的 $R$-(3,3'-二苯基-1, $1^{\prime}$-二䒺基)-20-冠-6 冠醚固定相 $(\mathrm{CSP})$. 在以 $\mathrm{pH}=2$ 的高氯酸溶液为流动相, 流速为 $0.1 \mathrm{~mL} \cdot \mathrm{min}^{-1}$, 柱温为 $25{ }^{\circ} \mathrm{C}$ 的条件下, 研究了 $R-\left(3,3^{\prime}-\right.$ 二苯基-1,1'-二䒬基)-20-冠- 6 冠醚固定相(CSP)对 13 种 $\alpha$-氨基酸对映体的拆分能力, 并将其拆分结果与商品 CR $(+$ )柱进 行了对比. 实验结果表明在此色谱条件下, 有 11 种 $\alpha$-氨基酸对映体(苯甘氨酸、对羟基苯甘氨酸、蛋氨酸、酪氨酸、色 氨酸、顿氨酸、亮氨酸、异亮氨酸、苯丙氨酸、谷氨酸、天冬氨酸)能得到不同程度的拆分，并且对其中 6 种 $\alpha$-氨基酸 对映体的拆分效果要好于商品 $\mathrm{CR}(+)$ 柱. 研究表明此冠醚手性固定相对 $\alpha$-氨基酸对映体有很好的手性识别能力, 在对 $\alpha$-氨基酸对映体的拆分上能与商品 $\mathrm{CR}(+)$ 柱相謧美.
\end{abstract}

关键词 $\alpha$-氨基酸对映体; 冠醚; 手性固定相; 手性识别; 高效液相色谱

\section{Synthesis and Evaluation of Crown Ether Chiral Stationary Phase}

\author{
Lu, Zhenyu Wu, Peng Zi, Min Yang, Canyu Kong, jiao Yuan, Liming* \\ (Faculty of Chemistry and Chemical Engineering, Yunnan Normal University, Kunming 650500)
}

\begin{abstract}
In this paper, $R$-(3,3'-diphenyl-1,1'-binaphthyl)-20-crown-6 was synthesized from $(R)-1,1^{\prime}$-bi(2-naphthol), which was coated on $\mathrm{C}^{18}$ silica gel (the average particle size is $5 \mu \mathrm{m}$, and aperture is $120 \AA$ ). The final product (CSP) was used as the chiral stationary phase of high performance liquid chromatography for the enantioseparation of $\alpha$-amino acids. In the conditions of perchloric acid solution $(\mathrm{pH}=2)$ as mobile phase, flow rate of $0.1 \mathrm{~mL} \cdot \mathrm{min}^{-1}, 25{ }^{\circ} \mathrm{C}$, the chiral recognition ability for 13 kinds of $\alpha$-amino acids enantiomer on CSP was studied, and 11 kinds of $\alpha$-amino acid enantiomers (phenylglycine, hydroxyphenylglycine, methionine, tyrosine, tryptophan, valine, leucine, isoleucine, phenylalanine, glutamate, aspartate) were separated. This column offers a better enantioselectivity for six kinds of $\alpha$-amino acid than that of commercial CR $(+)$ column. The experimental results showed that CSP possesses a good enantioselectivity for $\alpha$-amino acid enantiomers.

Keywords $\alpha$-amino acid enantiomers; crown ethers; chiral stationary phase; chiral recognition; high performance liquid chromatography
\end{abstract}

冠醚是具有空腔的大环聚醚，这类化合物呈现王冠 状结构, 其环的外沿是亲脂性的乙撑基, 环的内沿是富 电子的杂原子, 如 O, N, S 等. 1967 年, Pederson 发现冠 醚后 ${ }^{[1]}, 18$-冠-6-醚就常用于高效液相色谱分离化合物 ${ }^{[2]}$, 但 18-冠-6-梄没有手性, 要在其分子中引入手性中心后 才能作为手性识别剂, 而 1,1'-联菜单体是最常用的一种 手性识别剂. 18-冠-6-醚中引入一个 1,1'-联䒺单体能较 好地用于液相色谱法拆分外消旋 $\alpha$-氨基酸 ${ }^{[3 \sim 6]}$. 在众多 的冠醚手性固定相中, (3,3'-二苯基-1,1'-二菜基)-20-冠-6 表现出极好的拆分能力 ${ }^{[7,8]}$. 但目前国内还未见对
$R$-(3,3'-二苯基-1, $1^{\prime}$-二菜基)-20-冠-6 的合成及将其涂敷 到 $\mathrm{C}^{18}$ 硅胶做成手性固定相的报道，该文利用铃木反应 等对国外已报道的该手性材料的合成路线进行了改 进 ${ }^{[8]}$, 以 $R$-联芸酚为原料经过 5 步反应合成了 $R$ - $\left(3,3^{\prime}-\right.$ 二苯基-1,1'-二䒺基)-20-冠- 6 , 使反应产率大大提高. 将 $R$-(3,3'-二苯基-1, $1^{\prime}$-二菜基)-20-冠- 6 涂敷于 $5 \mu \mathrm{m}$ 的 $\mathrm{C}^{18}$ 硅胶上制得了 $R$-(3,3'-二苯基-1,1'-二荎基)-20-冠- 6 手性 固定相, 并研究了其对 13 种 $\alpha$-氨基酸对映体的识别能 力, 取得了满意的效果.

$R$-(3,3'-二苯基-1, $1^{\prime}$-二萗基)-20-冠-6 的合成路线如

\footnotetext{
* E-mail: yuan_limingpd@126.com

Received August 11,2014; revised September 23, 2014; published online October 9, 2014.

Project supported by the National Natural Science Foundation of China (Nos. 21275126, 21127012).

国家自然科学基金(Nos. 21275126, 21127012)资助项目.
} 
Scheme 1 所示.

\section{1 结果与讨论}

\section{$1.1 R$-(3,3'-二苯基-1, ' $^{\prime}$-二萗基)-20-冠-6 构型的测试}

在高效液相色谱中, 用商品 CHIRALCEL OD-H 柱 拆分、紫外检测器和 CHIRALYSER 旋光检测器串联检 测, 流动相为正己烷/异丙醇 $(V / V=9 / 1)$, 流速为 0.5 $\mathrm{mL} \cdot \mathrm{min}^{-1}$, 柱温为 $25{ }^{\circ} \mathrm{C}$ 的条件下测 $R$-(3,3'-二苯基-1, $1^{\prime}$ 二菜基)-20-冠-6. 图 1(a)为商品 OD 柱分离后测得的紫 外检测器峰, 从中我们可以看出在 $15 \mathrm{~min}$ 前有一个很强 的正峰, 图 1(b)为 CHIRALYSER 旋光检测器上测得的 谱图, 因为是串联检测, 样品后经过旋光检测器, 所以 从图 1 可以看出在 $15 \mathrm{~min}$ 后有一个很强的倒峰, 说明在 合成 $R$-(3,3'-二苯基-1, $1^{\prime}$-二䒺基)-20-冠-6 的过程中, 构 型基本保持不变.

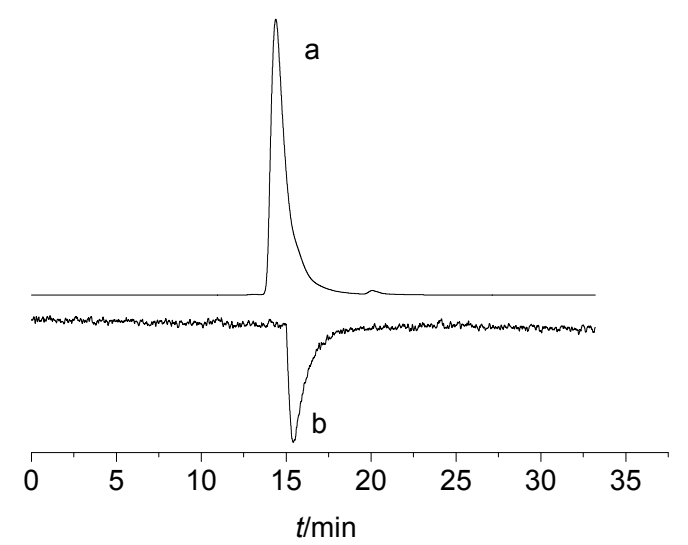

图 1 手性 HPLC 拆分 $R$-(3,3'-二苯基-1,1'-二䒬基)-20-冠-6 谱 图

Figure 1 Chromatograms of $R$-(3,3'-diphenyl-1,1'-binaphthyl)20-crown- 6 by chiral HPLC
$1.2 R$-(3,3'-二苯基-1, $1^{\prime}$-二萗基)-20-冠-6 合成路线的 改进

文献[8]报道了在合成中间产物 $R-3,3^{\prime}$-苯基-2,2'-二 甲氧基-1, $1^{\prime}$-二䒺的过程中，采用格氏试剂来取代苯环 上的溴, 其操作条件要求绝对无水无氧, 十分苛刻, 并 且其产率不高. 与文献 [8]相比，本工作采用铃木反应， 此反应条件简单只需要除去空气，不需要绝对无水条 件, 使反应易进行, 并且铃木反应中用到的催化剂 $\left[1,1^{\prime}-\right.$ 双 (二苯基膦)二茂铁 $]$ 二氯化钯 $\left[\mathrm{PdCl}_{2}(\mathrm{dppf})\right]$ 催化效率高, 副产物少，使这一步的产率直接从 $38 \%$ 提高到 $85 \%$. 在 最后一步 $R$-(3,3'-二苯基-1, $1^{\prime}$-二菜基)-20-冠-6 的纯化过 程中，文献[8]用柱色谱后再次凝胶柱色谱的方法，凝胶 柱中所使用的凝胶价格十分昂贵. 与文献[8]相比, 本工 作采用柱色谱后用正庚烷重结晶的方法，同样可得到很 纯的冠醚产物. 正庚烷分析纯跟凝胶相比便宜很多, 可 以显著地降低合成成本.

\section{$1.3 R$-(3,3'-二苯基-1, $1^{\prime}$-二萗基)-20-冠-6 手性固定相 的元素分析}

对 $\mathrm{C}^{18}$ 硅胶和 $R$-(3,3'-二苯基-1,1'-二菜基)-20-冠-6 手性固定相(CSP)进行元素分析, 结果见表 1. 根据元素 分析的结果可以算出 $R$-(3,3'-二苯基-1, $1^{\prime}$-二䒺基)-20-冠6 的涂敷质量为 $\mathrm{C}^{18}$ 硅胶质量的 $9.35 \%$, 其与我们的实际 值 $10 \%$ 基本一致.

表 1 元素分析结果

Table 1 The results of elemental analysis

\begin{tabular}{cccc}
\hline No. & $\mathrm{N} / \%$ & $\mathrm{C} / \%$ & $\mathrm{H} / \%$ \\
\hline $\mathrm{C}^{18}$ 硅胶 & $<0.5$ & 16.84 & 3.19 \\
$\mathrm{CSP}$ & $<0.5$ & 23.44 & 3.65 \\
\hline
\end{tabular}<smiles>Oc1ccc2ccccc2c1-c1c(O)ccc2ccccc12</smiles>

A<smiles>COc1c(-c2ccccc2)cc2ccccc2c1-c1cc2ccccc2c(OC)c1-c1ccccc1</smiles>

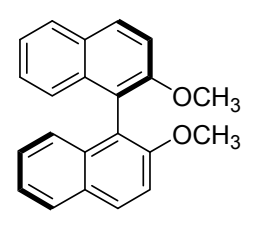

B

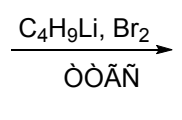<smiles>Oc1c(-c2ccccc2)cc2ccccc2c1-c1c(O)c(-c2ccccc2)cc2ccccc12</smiles><smiles>COc1c(Br)cc(Br)cc1-c1c(OC)c(Br)cc2ccccc12</smiles>

C

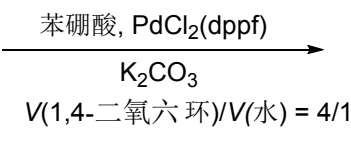

$V(1,4-$ 二氧六环 $) / V($ 水 $)=4 / 1$

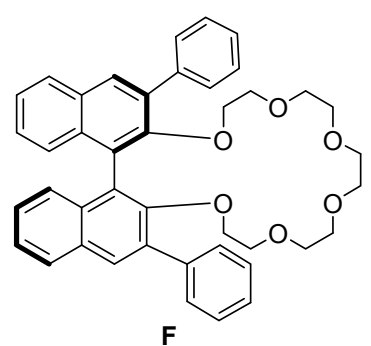

图式 1 合成路线

Scheme 1 Synthetic route 
$1.4 R$-(3,3'-二苯基-1,1'-二萗基)-20-冠-6 手性柱柱效 与峰形

以 $L$-对羟基苯甘氨酸测柱效, 计算塔板数为: $n=$ $5.54 \times\left(t_{1} / w_{1 / 2}\right)^{2}=57638$ 块 $/ m$ ( $t_{1}$ 出峰时间、 $w_{1 / 2}$ 为半峰 高). 实际塔板数为 57638 块 $/ m$, 说明柱效还是比较好 的.

不对称因子: $A_{\mathrm{s}}=B / A=1.51$ ( $A$ 为 $10 \%$ 峰高处前半 峰的宽度、 $B$ 为同高度处后半峰的宽度). 算出来不对称 因子 $A_{\mathrm{s}}$ 为 1.51 , 说明峰形还是比较好.

\section{$1.5 R$-(3,3'-二苯基-1, $1^{\prime}$-二萗基)-20-冠-6 手性柱对 $\alpha$ - 氨基酸对映体的拆分}

将 $R$-(3, $3^{\prime}$-二苯基-1, $1^{\prime}$-二芸基)-20-冠-6 涂敷型手性 柱, 在 $\mathrm{pH}=2$ 的高氯酸溶液作流动相, 流速为 0.1 $\mathrm{mL} \cdot \mathrm{min}^{-1}$, 柱温为 $25{ }^{\circ} \mathrm{C}$ 的条件下拆分 13 种常见的 $\alpha$ 氨基酸对映体: 苯甘氨酸(1)、对羟基苯甘氨酸(2)、蛋氨 酸 $(3)$ 、酪氨酸 $(4)$ 、苯丙氨酸 $(5)$ 、精氨酸 $(6)$ 、色氨酸 $(7)$ 、 顿氨酸 $(8)$ 、异亮氨酸 $(9)$ 、亮氨酸 $(10)$ 、组氨酸 $(11)$ 、谷 氨酸(12)、天冬氨酸(13).

\section{6 拆分情况}

表 2 列出了氨基酸在手性柱上的拆分, 并将它与商 品柱和相关文献进行了比较.

保留因子 $k_{1}=\left(t_{1}-t_{0}\right) / t_{0}, k_{2}=\left(t_{2}-t_{0}\right) / t_{0}$; 分离因子 $\alpha=k_{2} / k_{1}$; 分离度 $R_{\mathrm{s}}=1.18\left(t_{2}-t_{1}\right) /\left[W_{1 / 2}(1)+W_{1 / 2}(2)\right]$; 其 中 $t_{1}, t_{2}$ 分别为样品第一个流出峰和第二个流出峰的保 留时间; $W_{1 / 2}(1), W_{1 / 2}$ (2) 分别为两个对映体色谱峰的半峰 宽.

从表 2 中可以看出, 在拆分的 13 个 $\alpha$-氨基酸对映 体中 1 5 和 7 12 这 11 种能得到分离, 分离谱图如图
2 所示. 在能够分离的 11 种 $\alpha$-氨基酸对映体中拆分效果 比较好的是 $1\left(\alpha=3.74, R_{\mathrm{s}}=9.18\right), 2\left(\alpha=6.24, R_{\mathrm{s}}=\right.$ 18.26), $3\left(\alpha=1.80, R_{\mathrm{s}}=2.81\right), 7\left(\alpha=1.86, R_{\mathrm{s}}=1.02\right), 8$ $\left(\alpha=2.57, R_{\mathrm{s}}=1.97\right), 10\left(\alpha=1.59, R_{\mathrm{s}}=1.62\right), 12(\alpha=2.38$, $\left.R_{\mathrm{S}}=3.39\right), 13\left(\alpha=6.35, R_{\mathrm{S}}=6.48\right)$, 这 8 种 $\alpha$-氨基酸对映 体都能得到基线分离.

与商品 $\mathrm{CR}(+)$ 柱相比, 本色谱柱具有一定的优势. 商品 $\mathrm{CR}(+)$ 柱分离 $1,2,5 \sim 7$ 样品有着较好的手性分离 能力, 但是在分离 $4,8 \sim 10,12,13$ 样品上, 商品 $\mathrm{CR}(+)$ 柱的分离度 $R_{\mathrm{s}}$ 小于本文研究冠梄柱, 说明在分离这 6 种 氨基酸对映体上，商品 $\mathrm{CR}(+)$ 柱没有本文色谱柱手性 分离能力好, 更重要的是缬氨酸 $(8)$ 在本色谱柱上得到 了分离, 但是在商品柱上没有分离. 与文献报道相比, 本色谱柱在分离 $4,8,13$ 上也具有优势. 造成差异的主 要原因是 3 种柱的尺寸不一致, 另外手工制柱的再现性 也相对较差, 还有就是所用 $\mathrm{C}^{18}$ 的不同也是造成不一致 的原因. 但它们总的对氨基酸的拆分趋势是一致的.

$\alpha$-氨基酸对映体在冠醚手性固定相上保留时间的 不同可以这样来解释 ${ }^{[8]}$ : 氨基酸上的氨基在流动相高氯 酸溶液中会被质子化转变成氨基酸阳离子，该阳离子会 分布于流动相中, 并和固相相手性冠梄环中的氧原子作 用. 由于冠醚中的手性联菜酚造成的空间位阻，氨基酸 的 $D$-及 $L$-构型与冠醚环的作用力不同, 使得氨基酸外 消旋体在手性柱中的移动速度不同，因此氨基酸的外消 旋体在手性冠醚柱中得到手性拆分.

\section{7 典型的拆分谱图}

图 2 是一些 $\alpha$-氨基酸对映体的拆分谱图, 从这些图 谱可见这些 $\alpha$-氨基酸对映体都得到了很好的分离, 其中 大多数都得到了基线分离.

表 2 手性柱对 $\alpha$-氨基酸对映体的拆分结果

Table 2 The separation results of $\alpha$-amino acid enantiomers on chiral columns

\begin{tabular}{|c|c|c|c|c|c|c|c|c|c|c|c|c|}
\hline \multirow{2}{*}{ 对映体 } & \multicolumn{4}{|c|}{ 色谱柱 ${ }^{a}$} & \multicolumn{4}{|c|}{ 商品 $\mathrm{CR}(+)$ 柱 ${ }^{b}$} & \multicolumn{4}{|c|}{ 文献报道 ${ }^{[7] c}$} \\
\hline & $k_{1}^{\prime}$ & $k_{2}^{\prime}$ & $\alpha$ & $R_{\mathrm{S}}$ & $k_{1}^{\prime}$ & $k_{2}^{\prime}$ & $\alpha$ & $R_{\mathrm{S}}$ & $k_{1}^{\prime}$ & $k_{2}^{\prime}$ & $\alpha$ & $R_{\mathrm{S}}$ \\
\hline 1 & 2.29 & 8.58 & 3.74 & 9.28 & 1.21 & 5.25 & 4.31 & 8.52 & 2.00 & 9.17 & 4.58 & 13.66 \\
\hline 2 & 1.17 & 7.36 & 6.24 & 18.62 & 0.74 & 5.36 & 7.27 & 14.60 & $\underline{e}^{e}$ & $-^{e}$ & $\underline{-}^{e}$ & $\underline{-e}^{e}$ \\
\hline 3 & 1.86 & 3.34 & 1.80 & 2.81 & 0.87 & 1.85 & 2.12 & 3.28 & 1.77 & 3.65 & 2.06 & 6.66 \\
\hline 4 & 6.64 & 8.39 & 1.26 & 3.13 & 2.19 & 3.12 & 1.42 & 5.83 & 7.11 & 8.61 & 1.21 & 2.64 \\
\hline 5 & 8.64 & 10.95 & 1.27 & 1.20 & 3.03 & 4.18 & 1.37 & 1.13 & 9.25 & 11.50 & 1.24 & 2.95 \\
\hline 6 & 0.49 & 0.49 & 1.00 & $-^{d}$ & 0.18 & 0.26 & 1.45 & 0.25 & 0.36 & 0.62 & 1.75 & 1.77 \\
\hline 7 & 8.19 & 15.30 & 1.86 & 1.02 & 18.04 & 22.66 & 1.25 & 1.48 & $\underline{e}^{e}$ & $\underline{e}^{e}$ & $\underline{-}^{e}$ & $\underline{-e}^{e}$ \\
\hline 8 & 0.39 & 1.00 & 2.57 & 1.97 & 0.34 & 0.34 & 1.00 & $-^{d}$ & 0.89 & 1.01 & 1.14 & 0.66 \\
\hline 9 & 2.63 & 3.03 & 1.15 & 0.95 & 0.85 & 1.07 & 1.26 & 0.34 & 2.44 & 2.88 & 1.18 & 1.62 \\
\hline 10 & 2.60 & 4.14 & 1.59 & 1.62 & 1.15 & 2.05 & 1.91 & 1.26 & 3.11 & 5.14 & 1.65 & 5.73 \\
\hline 11 & 0.38 & 0.38 & 1.00 & $\smile^{d}$ & 0.17 & 0.17 & 1.00 & $\complement^{d}$ & 0.21 & 0.21 & 1.00 & $\simeq^{d}$ \\
\hline 12 & 0.46 & 1.09 & 2.38 & 3.39 & 0.03 & 0.48 & 6.00 & 1.80 & 0.32 & 1.18 & 3.73 & 4.94 \\
\hline 13 & 0.32 & 2.04 & 6.35 & 6.48 & 0.18 & 0.72 & 3.96 & 2.79 & 0.14 & 0.28 & 2.06 & 0.72 \\
\hline
\end{tabular}

色谱条件: 流动相为 $\mathrm{pH}=2$ 的高氯酸, 流速为 $0.1 \mathrm{~mL} \cdot \mathrm{min}^{-1}$, 柱温为 $25{ }^{\circ} \mathrm{C}$; 柱规格: $250 \mathrm{~mm} \times 2 \mathrm{~mm} .{ }^{b}$ 色谱条件: 流动相为 $\mathrm{pH}=2$ 的高氯酸, 流速为 0.4 $\mathrm{mL} \cdot \mathrm{min}^{-1}$, 柱温为 $25{ }^{\circ} \mathrm{C}$; 柱规格: $150 \mathrm{~mm} \times 4 \mathrm{~mm}$. ${ }^{\mathrm{C}}$ 色谱条件: 流动相为 $\mathrm{pH}=2$ 的高氯酸, 流速为 $0.5 \mathrm{~mL} \cdot \mathrm{min}^{-1}$, 柱温为 $18{ }^{\circ} \mathrm{C}$; 柱规格: $125 \mathrm{~mm} \times 4 \mathrm{~mm}$; ${ }^{d}$ 未分开. ${ }^{e}$ 文献未报道. 

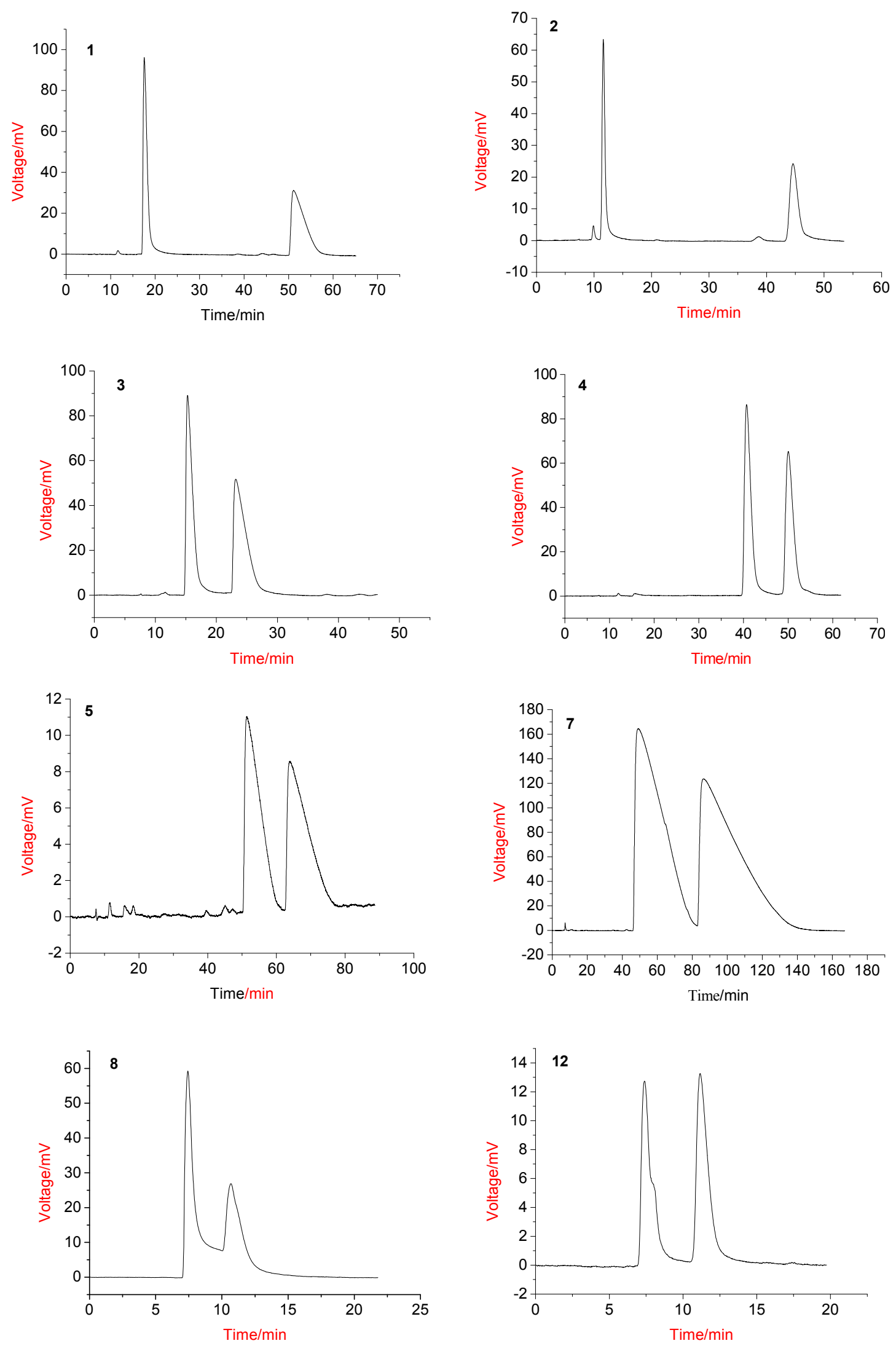

图 $2 \alpha$-氨基酸对映体的拆分谱图

Figure 2 Enantioseparation chromatograms of $\alpha$-amino acid 
$1.8 R$-(3,3'-二苯基-1, $1^{\prime}$-二萗基)-20-冠-6 手性固定相 涂敷质量的影响

在制备 $R$-(3,3'-二苯基-1, $1^{\prime}$-二䒬基)-20-冠-6 冠醚固 定相(CSP)时, 分别制备了涂敷冠醚质量与 $\mathrm{C}^{18}$ 硅胶质量 比分别为 5\%, 10\%, 15\%的 3 种手性固定相. 实验结果表 明: 当冠醚涂敷质量比为 $5 \%$ 时, 由于冠醚量少会使氨 基酸与冠醚接触的位点比较少, 使其分离效果大大下 降; 当冠醚涂敷质量比为 $10 \%$ 和 $15 \%$ 时拆分效果比较 好, 从最终产物冠醚合成不易和节约成本的方面考虑所 以选择 $10 \%$ 的涂敷量.

\section{$1.9 R$-(3,3'-二苯基-1,1'-二萗基)-20-冠-6 手性固定相 的稳定性}

该固定相在 $\mathrm{pH}=2$ 的高氯酸溶液为流动相的条件 下使用 $360 \mathrm{~h}$ 后, 各 $\alpha$-氨基酸的容量因子基本保持不变, 并且测定该固定相的含碳量也几乎没有改变. 结果表明 $R$-(3,3'-二苯基-1, 1'-二䒺基)-20-冠-6 手性固定相化学稳 定性良好, 在 $\mathrm{pH}=2$ 的酸性条件下可以长时间的使用.

\section{2 结论}

以 $R$-联菜酚为原料, 利用铃木反应将文献报道合 成路线进行改进, 合成了 $R$ - $\left(3,3^{\prime}\right.$-二苯基-1, ' ' - 二萗 基)-20-冠-6, 使反应条件简单易行, 大大提高了反应产 率. 将其涂敷在 $5 \mu \mathrm{m}$ 的 $\mathrm{C}^{18}$ 硅胶上制成了高效液相色谱 冠醚手性固定相, 以 $\mathrm{pH}=2$ 的高氯酸为流动相, 对 13 种常见的 $\alpha$-氨基酸对映体进行了拆分, 并与商品 $\mathrm{CR}(+)$ 柱和文献报道 ${ }^{[7]}$ 的拆分结果做了对比. 结果表明 11 种 $\alpha$-氨基酸对映体能得到分离, 并且有 8 种 $\alpha$-氨基酸 对映体能达到基线分离, 其中对 6 种氨基酸拆分效果要 好于商品 $\mathrm{CR}(+)$ 柱, 说明本文以 $R-\left(3,3^{\prime}\right.$-二苯基-1,1'-二 芸基)-20-冠-6 为材料的手性固定相在分离这些 $\alpha$-氨基 酸对映体有着相当好的效果.

\section{3 实验部分}

\section{1 仪器与试剂}

DF-101S 集热式恒温加热磁力搅拌器(巩义市予华 仪器有限公司); RE5298A 型旋转蒸发仪(上海亚荣生化 仪器厂); Bruker-500 核磁共振仪; LC600 高效液相色谱 仪(美国, Labtech); XT-4 双目显微熔点测定仪(北京泰克 仪器有限公司); 色谱工作站(美国, Labtech); 紫外波长 检测器(美国, Labtech); 液相色谱装柱机(美国, Alltech), 不锈钢空色谱柱: $250 \mathrm{~mm} \times 2.0 \mathrm{~mm}$ i.d.(美国, Alltech); VarioEL III 元素分析仪(德国); CHIRALYSER-MP 旋光 检测器(德国); 商品手性柱: $150 \mathrm{~mm} \times 4.0 \mathrm{~mm}$ (大赛璐药 物手性技术有限公司).
$R$-联萗酚(大赛璐); $\mathrm{CH}_{3} \mathrm{I}$ (西亚); $n$-丁基锂(adamas); 苯嗍酸(adamas); $\mathrm{BBr}_{3}$ (adamas); [1,1'-双(二苯基膦)二茂 铁]二氯化钯 $\left[\mathrm{PdCl}_{2}(\mathrm{dppf})\right]$ (adamas); 二对甲苯磺酸戊乙 二醇(Alfa); 球型 C-18 硅胶(美国赛分科技有限公司，粒 径 $5 \mu \mathrm{m}$, 孔径 $120 \AA$ ); 其余试剂均为国产的分析纯.

\section{$3.22,2^{\prime}$-二甲氧基-1,1'-二萗(B)的合成}

取 $R$-联萗酚 $10 \mathrm{~g}$ 加入 $180 \mathrm{~mL}$ 丙酮中, 加入 $26 \mathrm{~g}$ $\mathrm{K}_{2} \mathrm{CO}_{3}$ 作催化剂, 在 $60{ }^{\circ} \mathrm{C}$ 磁力回流摚拌, 在回流过程 中缓慢滴加碘甲烷 $6.52 \mathrm{~mL}$, 反应 $4 \mathrm{~h}$. 混合物用二氯甲 烷萃取, 有机层用无水 $\mathrm{MgSO}_{4}$ 干燥和蒸发. 然后用硅胶 柱纯化[洗脱剂 $V$ (乙酸乙酯) $: V$ (石油醚) $=1: 5$ ], 减压 除去洗脱剂得白色晶体 $\mathbf{B}^{[9]} 10.54 \mathrm{~g}$, 产率 $96 \%$. m.p. $188 \sim 191{ }^{\circ} \mathrm{C}$ (文献值 ${ }^{[10]}: 190 \sim 191{ }^{\circ} \mathrm{C}$ ); ${ }^{1} \mathrm{H}$ NMR $\left(\mathrm{CDCl}_{3}, 500 \mathrm{MHz}\right) \delta: 8.00$ (d, $\left.J=9.0 \mathrm{~Hz}, 2 \mathrm{H}, \mathrm{ArH}\right), 7.89$ (d, $J=8.2 \mathrm{~Hz}, 2 \mathrm{H}, \mathrm{Ar}-\mathrm{H}), 7.49$ (d, $J=9.0 \mathrm{~Hz}, 2 \mathrm{H}, \mathrm{Ar}-\mathrm{H})$, 7.34 (t, $J=7.4 \mathrm{~Hz}, 1 \mathrm{H}, \operatorname{Ar}-\mathrm{H}), 7.23(\mathrm{t}, J=7.3 \mathrm{~Hz}, 1 \mathrm{H}$, Ar-H), 7.13 (d, $J=8.5 \mathrm{~Hz}, 2 \mathrm{H}, \operatorname{Ar}-\mathrm{H}), 3.79$ (s, 6H, $\left.\mathrm{Ar}-\mathrm{OCH}_{3}\right)$.

\section{$3.33,3^{\prime}$-二溴基-2,2'-二甲氧基-1,1'-二萗(C)的合成}

在氮气的保护下，将 $37.6 \mathrm{~mL}$ 的 $n-\mathrm{C}_{4} \mathrm{H}_{9} \mathrm{Li}(1.6$ $\mathrm{mol} / \mathrm{L}$ )加入到四甲基二乙胺 $(6.1 \mathrm{~mL})$ 的 $200 \mathrm{~mL}$ 乙醚溶液 中, $25{ }^{\circ} \mathrm{C}$ 搅拌 $15 \mathrm{~min}$, 加入 $8.0 \mathrm{~g}$ 产物 $\mathbf{B}$ 搅拌 $3 \mathrm{~h}$, 然后 将反应物冷却到 $-75{ }^{\circ} \mathrm{C}$, 在 $10 \mathrm{~min}$ 内加入 $\mathrm{Br}_{2}(3.9 \mathrm{~mL})$ 的戊烷 $(20 \mathrm{~mL})$ 溶液, 然后将反应物升到室温摚拌过夜, 最后加入 $160 \mathrm{~mL}$ 饱和亚硫酸钠溶液摚拌 $4 \mathrm{~h}$. 混合物用 二氯甲烷萃取, 有机层用无水 $\mathrm{MgSO}_{4}$ 干燥和蒸发. 然后 用硅胶柱纯化 [洗脱剂 $V$ (丙酩) $: V$ (环己烷 $)=1: 40$ ], 减 压除去洗脱剂得淡黄色晶体 C $6.97 \mathrm{~g}$, 产率 58\%. m.p. $175 \sim 178{ }^{\circ} \mathrm{C}$ (文献值 ${ }^{[11]}: 178 \sim 182{ }^{\circ} \mathrm{C}$ ); ${ }^{1} \mathrm{H}$ NMR $\left(\mathrm{CDCl}_{3}, 500 \mathrm{MHz}\right) \delta: 8.30(\mathrm{~s}, 2 \mathrm{H}, \mathrm{ArH}), 7.85$ (d, $J=8.2$ $\mathrm{Hz}, 2 \mathrm{H}, \mathrm{ArH}), 7.45(\mathrm{t}, J=7 \mathrm{~Hz}, 2 \mathrm{H}, \mathrm{ArH}), 7.28 \sim 7.32(\mathrm{~m}$, $2 \mathrm{H}, \operatorname{ArH}), 7.10(\mathrm{~d}, J=8.5 \mathrm{~Hz}, 2 \mathrm{H}, \operatorname{ArH}), 3.53(\mathrm{~s}, 6 \mathrm{H}$, $\mathrm{ArOCH}_{3}$ ).

\section{$3.43,3^{\prime}$-苯基- $2,2^{\prime}$-二甲氧基-1, $1^{\prime}$-二萗(D)的合成}

在氮气保护下, 将 $6 \mathrm{~g}$ 产物 C, $6 \mathrm{~g}$ 苯硼酸和 $24 \mathrm{~g}$ $\mathrm{K}_{2} \mathrm{CO}_{3}$ 加入到 $160 \mathrm{~mL} 1,4$-二氧六环和水的混合溶液中 $[V(1,4$ 二氧六环 $): V($ 水 $)=4: 1]$, 再加入 $0.12 \mathrm{~g}$ $\mathrm{PdCl}_{2}$ (dppf)作为催化剂, 在 $110{ }^{\circ} \mathrm{C}$ 下回流摚拌 $24 \mathrm{~h}$. 混 合物用乙酸乙酯萃取, 有机层用 $\mathrm{MgSO}_{4}$ 干燥和蒸发. 然 后用硅胶柱纯化[洗脱剂 $V$ (乙酸乙酯) : $V$ (石油梄) $=1$ : $20]$, 减压除去洗脱剂得淡绿色油状固体 ${ }^{[12 ~ 15]} 5.03 \mathrm{~g}$, 产率 $85 \%$. ${ }^{1} \mathrm{H}$ NMR $\left(\mathrm{CDCl}_{3}, 500 \mathrm{MHz}\right) \delta: 8.01(\mathrm{~s}, 2 \mathrm{H}$, ArH), 7.95 (d, $J=8.2 \mathrm{~Hz}, 2 \mathrm{H}, \operatorname{ArH}), 7.81(\mathrm{~d}, J=5.0 \mathrm{~Hz}$, $4 \mathrm{H}, \mathrm{ArH}), 7.54 \sim 7.38(\mathrm{~m}, 8 \mathrm{H}, \mathrm{ArH}), 7.34 \sim 7.24(\mathrm{~m}, 4 \mathrm{H}$, 
Ar-H), 3.22 (s, 6H, Ar- $\mathrm{OCH}_{3}$ ).

\section{$3.53,3^{\prime}$-苯基-2,2'-二羟基-1,1'-二萗(E)的合成}

在氮气保护下, 将 $5 \mathrm{~g}$ 的产物 $\mathbf{D}$ 加入到 $150 \mathrm{~mL}$ 的 无水二氯甲烷溶液中, 将反应物降温到 $0{ }^{\circ} \mathrm{C}$ 缓慢地加 入 $10 \mathrm{~mL} \mathrm{BBr}_{3}$, 摚拌 $15 \mathrm{~min}$, 升温至 $25{ }^{\circ} \mathrm{C}$ 搅拌 $24 \mathrm{~h}$, 然 后再将反应物降温至 $0{ }^{\circ} \mathrm{C}$ 加水除去过量的 $\mathrm{BBr}_{3}$. 混合 物用二氯甲烷萃取, 有机层用 $\mathrm{MgSO}_{4}$ 干燥和蒸发. 然后 用硅胶柱纯化 [洗脱剂 $V$ (乙酸乙酯) : $V$ (石油醚 $)=1$ : $10]$, 减压除去洗脱剂得白色晶体 $3.90 \mathrm{~g}$, 产率 $83 \%$. m.p. $195 \sim 198{ }^{\circ} \mathrm{C}$ (文献值 ${ }^{[16]}$ : $197 \sim 199{ }^{\circ} \mathrm{C}$ ); ${ }^{1} \mathrm{H}$ NMR $\left(\mathrm{CDCl}_{3}, 500 \mathrm{MHz}\right) \delta: 8.05$ (s, 2H, $\left.\mathrm{ArH}\right), 7.95$ (d, $J=8.1$ $\mathrm{Hz}, 2 \mathrm{H}, \mathrm{ArH}), 7.77(\mathrm{~d}, J=5.1 \mathrm{~Hz}, 4 \mathrm{H}, \mathrm{ArH}), 7.53$ (t, $J=$ $15 \mathrm{~Hz}, 4 \mathrm{H}, \mathrm{ArH}), 7.49 \sim 7.32$ (m, 4H, ArH), 7.31 7.23 (m, 4H, ArH), 5.38 (s, 2H, Ar-OH).

\section{$3.6 R$-(3,3'-二苯基-1, $1^{\prime}$-二萗基)-20-冠-6 $(F)$ 的合成}

在氮气保护下, 将 $3 \mathrm{~g}$ 产物 $\mathbf{E}$ 和 $2.5 \mathrm{~g}$ 二对甲苯磺酸 戊乙二醇加入到 $180 \mathrm{~mL}$ 无水四氢呋喃溶液中, 再加入 $0.88 \mathrm{~g} \mathrm{KOH}$ 在 $60{ }^{\circ} \mathrm{C}$ 条件下搅拌回流 $72 \mathrm{~h}$. 混合物用二 氯甲烷萃取, 有机层用 $\mathrm{MgSO}_{4}$ 干燥和蒸发. 先用硅胶柱 纯化 [洗脱剂 $V$ (乙酸乙酯) $: ~ V$ (环己烷 $)=1: 2$ ]得黄色油 状固体, 然后再将其用正庚烷重结晶, 过滤, 得亮白色 晶体 $2.58 \mathrm{~g}$, 产率 $56 \%$. m.p. $68 \sim 72{ }^{\circ} \mathrm{C} ;{ }^{1} \mathrm{H}$ NMR $\left(\mathrm{CDCl}_{3}, 500 \mathrm{MHz}\right) \delta: 8.00$ (s, 2H, $\left.\mathrm{ArH}\right), 7.93$ (d, $J=8.1$ $\mathrm{Hz}, 2 \mathrm{H}, \mathrm{ArH}), 7.82$ (d, $J=7.5 \mathrm{~Hz}, 4 \mathrm{H}, \mathrm{ArH}), 7.49$ (t, $J=$ $7.5 \mathrm{~Hz}, 4 \mathrm{H}, \mathrm{ArH}), 7.44 \sim 7.39$ (m, 4H, ArH), $7.29 \sim 7.21$ (m, 4H, ArH), 3.76 3.72 (m, $\left.2 \mathrm{H}, \mathrm{CH}_{2} \mathrm{H}\right), 3.60(\mathrm{~s}, 4 \mathrm{H}$, $\left.\mathrm{CH}_{2} \mathrm{H}\right), 3.52 \sim 3.42\left(\mathrm{~m}, 6 \mathrm{H}, \mathrm{CH}_{2} \mathrm{H}\right), 3.40 \sim 3.29(\mathrm{~m}, 2 \mathrm{H}$, $\left.\mathrm{CH}_{2} \mathrm{H}\right), 3.25 \sim 3.21\left(\mathrm{~m}, 2 \mathrm{H}, \mathrm{CH}_{2} \mathrm{H}\right), 3.14 \sim 3.10(\mathrm{~m}, 2 \mathrm{H}$, $\left.\mathrm{CH}_{2} \mathrm{H}\right), 3.05 \sim 3.01\left(\mathrm{~m}, 2 \mathrm{H}, \mathrm{CH}_{2} \mathrm{H}\right)$.

\section{8 冠醚手性固定相的制备与装柱}

\subsection{1 手性固定相的涂敷}

取 $0.2 \mathrm{~g}$ 制得的 $R$-(3,3'-二苯基-1,1'-二菜基)-20-冠-6 于 $10 \mathrm{~mL}$ 二氯甲烷中, 振摇使其溶解. 按文献[17]的方 法将其涂敷于 $1.8 \mathrm{~g}$ 的 $5 \mu \mathrm{m}$ 的 $\mathrm{C}^{18}$ 硅胶上. 方法如下: 取冠醚溶液加到装有 $\mathrm{C}^{18}$ 硅胶的小烧瓶中, 使冠醚溶液 均匀分散到 $\mathrm{C}^{18}$ 硅胶上, 旋蒸除去溶剂, 将冠醚全部涂 覆在 $\mathrm{C}^{18}$ 表面上.

\subsection{2 柱子的装填}

采用湿法装柱, 取上述制备的手性固定相 $1.5 \mathrm{~g}$, 混 合于 $24 \mathrm{~mL}$ 甲醇/水 $(V / V=1 / 9)$ 溶液中, 搅拌成匀浆液,
用甲醇/水 $(V / V=1 / 9)$ 作顶替液, 在 $40 \mathrm{MP}$ 下进行装柱. 3.8 .3 色谱条件

流动相为 $\mathrm{pH}=2$ 的高氯酸溶液, 流速为 $0.1 \mathrm{~mL}$ • $\min ^{-1}$, 柱温为 $25{ }^{\circ} \mathrm{C}$. 流动相使用前均经过 $0.45 \mu \mathrm{m}$ 滤 膜过滤，超声脱气后备用; 检测波长为 $210 \mathrm{~nm}$.

辅助材料(Supporting Information) 化合物 $\mathbf{B} \sim \mathbf{F}$ 的 ${ }^{1} \mathrm{H}$ NMR 谱图. 这些材料可以免费从本刊网站(http://siocjournal.cn/)上下载.

\section{References}

[1] Pederson, C. J. Am. Chem. Soc. 1967, 89, 2495.

[2] Kiba, M. P.; Timbo, J. M.; Kaplan, L. J.; Jong, F.; Gokel, G. W.; Cram, D. J. J. Am. Chem. Soc. 1978, 100, 4555.

[3] Kyba, E. P.; Siegel, M. G.; Sousa, L. R.; Sogah, G. D. Y.; Cram, D. J. J. Am. Chem. Soc. 1973, 95, 2691.

[4] Yuan, L.-M. Chiral Recognition Materials, Science Press, Beijing, 2010, p. 149 (in Chinese). (袁黎明, 手性识别材料, 科学出版社, 北京, 2010, p. 149.)

[5] Sousa, L. R.; Sogah, G. D. Y.; Hoffman, D. H.; Cram, D. J. J. Am. Chem. Soc. 1978, 100, 4569.

[6] Sogah, G. D. Y.; Cram, D. J. J. Am. Chem. Soc. 1979, 101, 4569.

[7] Shinbo, T.; Yamaguchi, T.; Nishimura, K.; Sugiura, M. J. Chromatogr. 1987, 405, 145.

[8] Shinbo, T.; Yamaguchi, T.; Yanagishita, H.; Kitamoto, D.; Sakaki, K.; Sugiura, M. J. Am. Chem. Soc. 1992, 625, 101.

[9] Wang, F.; Li, W.-H.; Li, D.; Fan, Z.; Li, Z. Chin. J. Org. Chem. 2012, 32, 601 (in Chinese).

(王峰, 李稳宏, 李冬, 范峥, 李珍, 有机化学, 2012, 32, 601.)

[10] Alexander, M.; Andrew, G. T.; Derek, W. Y.; Edward, C. T. J. Am. Chem. Soc. 1980, 102, 6504.

[11] Martin, K.; Lars, R.; Sebastian, H.; Vijay, W.; Richard, G.; Benjamin, L. Synlett 2010, 2189.

[12] Chu, W.-Y.; Wang, M.; Li, X.-M.; Hou, Y.-J.; Sun, Z.-Z. Chin. J. Org. Chem. 2012, 32, 1666 (in Chinese).

(初文毅, 王熳, 李新民, 侯艳君, 孙志忠, 有机化学, 2012, 32, 1666.)

[13] Frank, S. A.; Chen, H.; Kunz, R. K.; Schnaderbeck, M. J.; R, W. R. Org. Lett. 2000, 2, 2691.

[14] Zou, Y.; Wang, Q.-R.; Tao, F.-G.; Ding, Z.-B. Chin. J. Chem. 2004, $22,215$.

[15] Liu, N.; Liu, C.; Jin, Z.-L. Chin. J. Org. Chem. 2012, 32, 860 (in Chinese).

(刘宁, 刘春, 金子林, 有机化学, 2012, 32, 860.)

[16] Yuya, O.; Shunichi, H.; Tadao, I.; Makoto, N. Tetrahedron 2005, $62,390$.

[17] Hou, S.-C.; Wang, M.; Zhou, Z.-Q.; Qiao, Z.; Guo, H.-C.; Shi, X.-Y. Chin. J. Chromatogr. 2002, 20, 537 (in Chinese). (候士聪, 王敏, 周志强, 乔振, 郭红超, 史雪岩, 色谱, 2002, 20, 537.) 